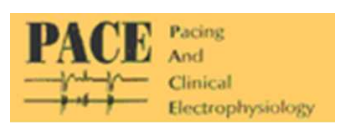

\title{
Association of Genetic Variation in Telomere Related SNP and Telomerase with Ventricular Arrhythmias in Ischaemic Cardiomyopathy
}

\begin{tabular}{|c|c|}
\hline Journal: & Pacing and Clinical Electrophysiology \\
\hline Manuscript ID & PACE-16-0364.R2 \\
\hline Wiley - Manuscript type: & Original - Devices \\
\hline Date Submitted by the Author: & $\mathrm{n} / \mathrm{a}$ \\
\hline Complete List of Authors: & $\begin{array}{l}\text { Sawhney, Vinit; St Bartholomew's Hospital, Cardiology } \\
\text { Brouilette, Scott; William Harvey Research Institute, TCT } \\
\text { Campbell, Niall; Barts Heart Centre, Cardiology } \\
\text { Coppen, Steven; William Harvey Research Institute, TCT } \\
\text { Baker, Victoria; Barts Heart Centre, Cardiology } \\
\text { Hunter, Ross; Barts Heart Centre, Cardiology } \\
\text { Dhinoja, Mehul; St Bartholomew's Hospital, Cardiology Research } \\
\text { Department } \\
\text { Johnston, Atholl; William Harvey Research Institute, TCT } \\
\text { Earley, Mark; Barts Heart Centre, Cardiology } \\
\text { Sporton, Simon; St Bartholomew's Hospital, Cardiology Research } \\
\text { Department } \\
\text { Suzuki, Ken; William Harvey Research Institute, TCT } \\
\text { Schilling, Richard; St Bartholomew's Hospital, Cardiology Research } \\
\text { Department }\end{array}$ \\
\hline Key Words: & Telomerase, TERC, SNP Genotyping, Ventricular Arrhythmia \\
\hline
\end{tabular}




\title{
ASSOCIATION OF GENETIC VARIATION IN TELOMERE RELATED SNP AND TELOMERASE WITH VENTRICULAR ARRHYTHMIAS IN ISCHAEMIC CARDIOMYOPATHY
}

Running Title: Sawhney et al; Telomere SNP variation and Arrhythmia

\begin{abstract}
*Sawhney Vinit MPhiL ${ }^{1,2}$, Brouilette Scott $\mathrm{PhD}^{2}$, Campbell Niall $\mathrm{PhD}^{1,2}$, Coppen Steven $\mathrm{PhD}^{2}$, Baker Victoria BSc${ }^{1}$, Hunter Ross $\mathrm{PhD}^{1}$, Dhinoja Mehul FRCP ${ }^{1}$, Johnston Atholl $\mathrm{PhD}^{2}$, Earley Mark $\mathrm{MD}^{1}$, Sporton Simon $\mathrm{MD}^{1}$, Suzuki Ken $\mathrm{PhD}^{2}$, Schilling Richard $M D^{1,2}$
\end{abstract}

1. Cardiology Department, St Bartholomew's Hospital, London, United Kingdom

2. William Harvey Research Institute, Queen Mary University, London, United Kingdom

*Corresponding Author: Vinit Sawhney

EP Cardiac Research

1 SMLG, $2^{\text {nd }}$ Floor East Wing

St Bartholomew's Hospital, London EC1A 7BE

Tel 442037658682

Fax 442034656483

Email: sawhneyvin@googlemail.com

Funding: this study was awarded a project grant by the Barts and the London Charity 


\section{ABSTRACT}

Background: Telomeres are known to provide genomic stability and telomere length has been associated with cardiovascular diseases. Moreover, a higher telomerase activity has been shown to be associated with ventricular arrhythmias (VA) in ischaemic cardiomyopathy. Increasing evidence suggests that genetic variation in key telomere genes has an impact on telomerase activity. Each copy of the minor allele of SNP rs12696304, at a locus including TERC (telomerase) has been associated with $\sim 75$ bp reduction in mean telomere length likely mediated by an effect on TERC expression. We investigated the impact of genetic variation of this SNP on telomerase and its association with VA in ischaemic cardiomyopathy patients.

Methods and Results: 90 ischaemic cardiomyopathy patients with primary prevention ICDs were recruited. 35 received appropriate ICD therapy for potentiallyfatal VA (cases) while the remaining 55 patients did not (controls). No significant differences in baseline demographics were seen between the groups. TS was measured by qPCR, telomerase activity by TRAP assay and SNP genotyping with Taqman probes. Telomerase was highest in $\mathrm{C}$ homozygous allele and had a significant association with VA in this group only (C/C,C/G,G/G; p-value 0.04, 0.33, $0.43)$.

Conclusion: The present study is the first to examine the association between telomerase, a SNP at a locus including TERC and VA in ischaemic cardiomyopathy patients. Homozygosity for C-allele significantly effects telomerase expression and its association with VA in this cohort. Large-scale prospective studies are required to determine if this genetic variation predisposes patients to greater arrhythmic tendency post MI.

Keywords: Telomerase, TERC, SNP genotyping, Ventricular arrhythmia 


\section{INTRODUCTION}

Telomeres are terminal sequences (TTAGGG) of linear eukaryotic chromosomes that are involved in maintaining genomic stability and regulating cellular proliferation. ${ }^{1}$ Telomeres shorten with every cell division, as DNA polymerase is not able to fully replicate the 3' end of the DNA strand. Progressive telomere shortening in somatic cells is a potential mechanism for a biological clock that determines cellular behaviour and is thought to trigger cell senescence and premature biological ageing. ${ }^{2}$ In addition to this replicative senescence, telomeres can undergo more stochastic shortening secondary to external stimuli including oxidative stress. ${ }^{3}$ Maintenance of telomere length is regulated by telomerase, a ribonucleoprotein enzyme consisting of a reverse transcriptase (TERT) and a RNA template (TERC) that facilitates addition of the telomere repeat sequence. ${ }^{4}$

Evidence suggests that some types of cardiac diseases represent a disease of premature biological ageing. Telomere length, independent of chronological age, has shown to be associated with coronary artery disease, ${ }^{5}$ premature myocardial infarction, ${ }^{6}$ poor renal function in heart failure, ${ }^{7}$ poor left ventricular ejection fraction ${ }^{8}$ and cardiovascular risk factors. ${ }^{9}$ More recently, our group has demonstrated that leukocyte telomere biology (critically short telomeres and telomerase activity) has a significant association with incidence of potentiallyfatal ventricular arrhythmia (VA) in patients with ischaemic cardiomyopathy. ${ }^{10}$ Moreover, there was no significant association between age, sex and WCC adjusted mean telomere length and ICD therapy for potentially-fatal VA ( $p$-value 0.88$){ }^{10}$

Telomere length has a strong genetic determination, with heritability estimates ranging from $44 \%$ to $80 \% .^{11,12}$ The telomere hypothesis postulates that shorter inherited telomeres could represent a primary abnormality predisposing to an increased risk of cardiovascular ageing. Since a substantial proportion of the marked inter-individual variation in telomere length is genetically determined, ${ }^{11,13,14}$ this should be taken into consideration when analysing inter-individual variability. The 
concept of genetics and heredity of telomere biology has emerged given the high inter-individual variation in telomere length across newborns ${ }^{15-18}$ and in individuals of the same age group, telomere length in poorly proliferating cells reflecting the telomere length found during early development, telomere length comparisons across monozygotic and dizygotic twins and the association of paternal age at conception and telomere length heritability. ${ }^{19}$ The vast majority of gene loci that have been associated with telomere length encode the TERT and TERC components of the enzyme telomerase. ${ }^{13,20}$ Increasing evidence suggests that genetic variation in key genes has an impact on telomerase activity. A genome wide association analysis of mean telomere length (in 2917 individuals with follow-up replication in 9492 individuals), identified an association between telomere length on 3q26 (rs 12696304, combined $p=3.72 \times 10^{-14}$ ) at a locus that includes TERC, which encodes the telomerase RNA component. Each copy of the minor allele of rs12696304 was associated with $\mathrm{a} \sim 75 \mathrm{bp}$ reduction in mean telomere length, equivalent to $\sim 3.6$ years of age related telomere length attrition. This association was thought to be mediated by an effect on TERC expression although telomerase activity was not measured. ${ }^{20}$

Thus genetically determined telomerase expression influences telomere length but its functional impact on somatic cells (where telomerase is largely suppressed) is unknown. Following on from our previous study demonstrating a significant association between telomere biology and $\mathrm{VA}^{10}$ and recent evidence suggesting genetic determination of telomere biology, it was hypothesised:

(a) Telomerase activity will be different across different genotypes of the SNP 12696304 on chromosome 3q26 and

(b) Differential telomerase activity across genotypes would have an impact on cardiovascular ageing and hence the incidence of VA in this cohort.

To test this hypothesis, the current case-control study was designed examining the association between genetic variation in SNP 12696304 and leukocyte 
telomere biology (telomerase activity and mean telomere length) with incidence of cardiac arrhythmia (VA) in ischaemic cardiomyopathy patients.

\section{METHODS}

\section{Study Design:}

Retrospective, cross sectional, case-control study. This study was approved by the local research ethics committee. All patients gave written informed consent. The study protocol conforms to the ethical guidelines of the 1975 Declaration of Helsinki as reflected in a priori approval by the institution's human research committee.

Patients were recruited at St Bartholomew's Hospital over one year. All laboratory telomere analysis and genotyping was carried out blinded to clinical data at William Harvey Research Institute.

\section{Subjects:}

Patients were included if they met the following criteria: (1) caucasian ethnicity, (2) previous MI with coronary artery disease demonstrated on angiography, (3) LVEF $\leq$ $35 \%$ and (4) ICD implanted for primary prevention (risk of SCD due to ischaemic cardiomyopathy). These criteria were used to improve uniformity across the patient group and to limit the effects of other causes of telomere length variation thereby representing the majority of the recruiting centre's patient population. ${ }^{21}$

Patients were excluded if they met any of the following criteria: (1) age $<18$ yrs, (2) chronic inflammatory disorders judged by patients' medical history, white cell count and serum CRP level, (3) carcinoma, (4) life expectancy less than 6 months as a result of non-cardiac disease and (5) inability to sign the consent form.

\section{Patient assessment and investigations:}

\section{$\underline{\text { Clinical }}$}

A standard questionnaire was filled in for all enrolled patients. Clinical details were obtained at recruitment by interviewing the patient and by examining their hospital 
records. The questionnaire recorded their medical history (to identify hypertension, diabetes, chronic renal failure, hypercholesterolemia, smoking status, family history of ischaemic heart disease and any other significant medical conditions), drug history, NYHA class, details of ICD implant, programming and previous arrhythmia history.

At recruitment, the patients also underwent a trans-thoracic echocardiogram to assess left ventricular function (LVEF measured by modified simpsons method), peripheral venous blood sample for general labs (including renal function, inflammatory markers - WCC and CRP, lipid profile), brain natriuretic peptide (BNP) and telomere genotyping.

Potentially-fatal VA was defined as sustained ventricular tachycardia $>170$ bpm or ventricular fibrillation, requiring device therapy.

\section{$\underline{\text { Telomere analysis }}$}

Telomere length and Telomerase activity

Whole blood was drawn into EDTA vacutainers and aliquots separated by density gradient centrifugation to yield concentrated leukocyte fraction (Buffy coat), which was used to isolate genomic DNA using QIAgen DNeasy Blood and Tissue Kits.

Using the isolated DNA, mean telomere length was measured by a modified version of the quantitative polymerase chain reaction (PCR) based technique that compares telomere repeat sequence copy number to single copy gene (36b4) copy number in a given sample. ${ }^{22-24}$ Samples were run in duplicate and a calibrator k562 run in all PCR reactions to allow comparison across runs.

Telomerase activity was assessed in whole leucocyte extracts obtained from thawed buffy coats using a commercially available standard PCR - ELISA kit (Roche). ${ }^{25}$ Each assay included telomerase positive control samples (included in the kit) and negative controls (heat inactivated samples). All samples were run in duplicate. 


\section{SNP Genotyping}

Taqman SNP genotyping and allelic discrimination were used to genotype patients to assess the association of genetic variation in telomere related SNP and ventricular arrhythmias.

All laboratory analyses were performed blinded to clinical data.

\section{Statistical Analyses:}

Statistical analyses were carried out using SAS VERSION 9.3 software. Continuous variables are reported as mean \pm standard deviation, or median (range) if not normally distributed. Characteristics of cases and controls were compared using chisquared test for categorical variables and unpaired t-test for continuous variables if normally distributed or Mann-Whitney $U$ test if not normally distributed. The effect of age, gender, WCC, genotype and other individual cardiovascular risk factors on telomere biology in individual genotypes was assessed using a logistic regression model. The individual genotype (telomere SNP rs 12636904), as a risk factor for appropriate ICD therapy for potential fatal VA (surrogate for SCD), alone and in combination with telomere biology (mean telomere length and every 1 unit increase in telomerase activity) was assessed using logistic regression.

\section{RESULTS}

\section{Study Patients:}

90 patients were enrolled in this study. Of these 35 were in the case arm (potentially-fatal VA) and 55 in the control arm (no potentially-fatal VA). There was no significant difference in the two groups in baseline demographics as shown in Table 1.

In the case arm, the vast majority of patients (93\%) had appropriate therapies for ventricular tachycardia. Only 2 out of the 35 cases had ventricular fibrillation that required ICD therapy. Both of these patients received therapy for ventricular 
tachycardia as well during the study follow-up period. The number of therapies for VA ranged from 1 to 12 (mean $\pm S D, 3.82 \pm 3.50$ ). Inappropriate therapy for rapidly conducted atrial fibrillation was seen in six patients. However, these patients also received ICD therapy for VAs and hence were included in the case arm of the study.

\section{Potentially-fatal VA and Genotype:}

There were 20 cases and 24 controls in the C/C genotype, 10 cases and 22 controls in $\mathrm{C} / \mathrm{G}$ genotype and 5 cases and 9 controls in the GG genotype. A univariate analysis examining the association of potentially-fatal VA and the genotype showed no significant association between the genotype and potentially-fatal VA (C/C OR 1; C/G OR 0.54, Cl 0.21 - 1.417, p-value 0.343; G/G OR 0.80, Cl 0.198 - 3.236, pvalue 0.907$)$.

An adjusted model, again showed no significant association between genotype and potentially-fatal VA (C/C OR 1; C/G OR 0.632, Cl $0.211-1.891$, pvalue 0.48; G/G OR 0.921, CI $0.194-4.374$, p-value 0.84) - Table 2.

\section{Potentially-fatal VA and Telomere Length in Different Genotypes:}

The mean telomere length was distributed normally and showed the expected age related attrition. The distribution of mean telomere length across different genotypes and its association with potentially-fatal VA in the individual genotypes was investigated.

There was no significant difference in mean telomere length between the different genotypes. Moreover, the mean telomere length had no association with potentially-fatal VA in the individual genotypes (C/C, C/G, G/G; p-value 0.58, 0.68, 0.73) - Table 3.

The mean telomere length, however, was shorter in individuals homozygous for $\mathrm{G}$ allele when compared to the heterozygotes and those homozygous for $\mathrm{C}$ allele 
$(C C>C G>G G$, mean \pm SD 1.25 $\pm 0.35,1.17 \pm 0.35,1.1 \pm 0.36 ; p$-value $C C>G G$, GG>CG, CG>CC; $0.17,0.53,0.32$ ). The difference in telomere lengths across genotypes did not achieve significance.

\section{Potentially-fatal VA and Telomerase in Different Genotypes:}

We investigated whether telomerase activity was different across genotypes and if there was an association between telomerase activity and genotype with potentiallyfatal VA.

In the entire cohort, the mean telomerase activity was highest in individuals homozygous for C-allele and was similar across the heterozygous allele and individuals homozygous for $\mathrm{G}$ allele (C/C 0.35, C/G 0.26, G/G 0.24).

The odds of receiving ICD therapy for potentially-fatal VA increase 7.5 fold in individuals homozygous for $\mathrm{C}$-allele with each unit increase in telomerase activity. There was a strong association between telomerase activity and ICD therapy in the C/C genotype (OR 7.507, Cl 1.00-56.61, p-value 0.04) - Table 4. This might not represent a statistically significant result due to the multiple testing issue of statistical analyses. However, the large effect size with an odds ratio greater than seven is notable and warrants further investigation.

No significant association between potentially-fatal VA and telomerase activity was found in the C/G genotype (OR 2.759, Cl $0.352-21.60$, p-value 0.33 ) or G/G genotype (OR 4.137, Cl 0.117 - 145.91, p-value 0.43) - Table 4.

Telomerase activity (mean $\pm S D$ ) in patients with and without potentially-fatal VA (cases versus controls) in different genotypes is shown in Figure 1. 


\section{DISCUSSION}

The present study is the first to examine the association between a SNP including the TERC locus, telomerase activity, and ventricular arrhythmias in ischaemic cardiomyopathy patients. The main finding of this study is that telomerase activity was much higher in individuals homozygous for C allele of SNP 12696304 and had a significant association with potentially-fatal VA in this genotype only. The odds of receiving ICD therapy for potentially-fatal VA increase 7.5 fold in individuals homozygous for C-allele with each unit increase in telomerase activity. This association with VA was not significant in G homozygous and heterozygous alleles. Hence homozygosity for C-allele significantly affected telomerase expression in ischaemic cardiomyopathy patients. Increased telomerase activity in individuals with $\mathrm{C} / \mathrm{C}$ genotype is associated with a higher incidence of VA post MI. Whether this association is causal or reverse-causal is not known. Also, the genotype (independent of the telomerase activity) did not correlate with incidence of VA.

Telomere length variability has been attributed to genetic influences. Heritability estimates of $78 \%$ have been derived from twin studies and account for inter-individual differences to a large extent. ${ }^{13}$ Large scale genotyping studies have identified various genetic variants which effect telomere length as they harbour genes that encode proteins with known function in telomere biology. These include TERC locus on $3 q 26,{ }^{20}$ TERT on 5p15.33, NAF1 (nuclear assembly factor 1 ) on 4q32.2, OBFC1 (oligonucleotide/oligosaccharide-binding fold containing 1) ${ }^{26}$ and RTEL 1 (regulator of telomere elongation helicase 1) on $20 q 13.3 .{ }^{27-30}$ TERC and TERT are components of the telomerase ribonucleoprotein moiety, whereas NAF1 is required for H/ACA box snoRNA assembly, the RNA family to which TERC belongs. ${ }^{27}$ Therefore, the three most significantly-associated loci $(3 q 26,5 p 15.33$ and 4q32.2) harbour genes involved in the formation and activity of telomerase. Hence, the genetic basis for this variation in telomere length is likely due to the presence of telomerase gene variants with variable activity in germ-line and/or stem cell pools. 
Of all the genetic loci associated with telomere length we studied the one with the most significant association - rs12696304, which is located $1.5 \mathrm{~kb}$ downstream of TERC and shown to be associated with mean telomere length. ${ }^{20}$ Our study did not show a significant difference in mean telomere length across the genotypes. This could be due to a small sample size. However, there was a trend towards shorter telomere length in individuals homozygous for $\mathrm{G}$ allele when compared to the heterozygotes and those homozygous for $\mathrm{C}$ allele. These findings showed a similar trend to those reported in other studies, although at a much smaller scale and in a different clinical cohort. ${ }^{22}$ In addition it was seen that the telomerase activity was highest in the C-homozygotes and lowest in the G-homozygotes. This points towards the likelihood of the observed genetic variation in telomere length to be mediated via TERC expression and may be validated in studies with a larger sample size.

As telomerase is responsible for maintaining telomere length, factors that influence telomerase activity are clearly important when considering the association between telomere length and clinical phenotype. However, significant associations between telomerase activity and SNP variants have not been shown in previous studies and all inferences have been derived from quantitative assessment of mean telomere length. The present study shows (1) a significant association between a telomere related SNP 12696304 and telomerase activity and (2) a significant association between telomerase activity and VA in individuals homozygous for the major allele (C) of the same SNP 12696304 post myocardial infarction. Thus, this genetic variant effects telomerase activity (and hence telomere length) and is significantly associated with the incidence of cardiac arrhythmia in individuals with ischaemic cardiomyopathy.

It is difficult to establish if the observed association is causal in nature within the realms of this cross-sectional study. The rise in telomerase activity could be the result of ICD therapy itself. Patients received ICD therapy for potentially fatal VA. Both the cardiac arrhythmia or ICD therapy itself could potentially affect cellular 
function and stability leading to an enzyme rise, which is independent of telomeric function. However, if this were the case, then one would expect the telomerase levels to be proportionate to the number of ICD therapies received. There was no significant association between telomerase activity and number of ICD therapies in this cohort $(n=90, p=0.653)$. Also, if the telomerase rise were solely the result of ICD therapy, then it would be difficult to explain why this association was seen predominantly in one genotype (homozygous C) despite it not including the patient with the maximum number of shocks. Nonetheless, conclusions can only be drawn with the availability of longitudinal changes in telomerase across all three alleles, before and after ICD therapy, from large-scale prospective studies.

If telomerase activity were to be causal in arrhythmia occurrence in this group of patients, then genetic variation in telomerase expression becomes clinically relevant. This genetic variation could predispose some individuals (C-homozygotes) to express higher telomerase activity at the time of index event (MI) in anticipation of later instability. This would mean that individuals being born with a certain predilection (mediated by telomere biology) would develop potentially-fatal VAs, should they suffer from a MI.

In this scenario, the increase in telomerase activity in individuals with $\mathrm{C}$ homozygous alleles of the SNP encoding TERC would be present prior to the development of VA. The increased telomerase expression in these individuals might be secondary to an inherited variation in TERC, which only becomes functional (increased telomerase activity) on exposure to inflammation and oxidative stress at the time of MI. The role of telomerase in arrhythmia prediction here may reflect a telomere independent role (driven by compromised telomeric integrity in Chomozygotes rather than telomere length alone) as a transcription factor driving expression of key mitochondrial genes to protect the mitochondrial genome from oxidative stress at the time of myocardial infarct. 
Poor left ventricular function and $\mathrm{Ml}$ are categorical traits, which might change with age or during the course of a disease process like coronary artery disease. However, genetic variation in TERC/telomerase expression would be present from birth and might become functional at the time of index $\mathrm{Ml}$ - before (or after) the development of left ventricular dysfunction or cardiac arrhythmias. These can thus be accurately quantified early on in the disease process. Thus, if increased telomerase activity is present in C homozygous alleles of the studied SNP, before development of left ventricular dysfunction post $\mathrm{MI}$, then this can be developed as an intermediate phenotype to identify individuals prone to VA relatively early on post MI. Again, this needs prospective studies to test the validity of the observed associations in this study.

\section{STUDY LIMITATIONS}

Some limitations of this study require consideration.

Due to the small sample size and absence of longitudinal data it is difficult to speculate the clinical relevance of genetic variation in telomerase expression and its association with cardiac arrhythmia in this cohort. Hence these findings are hypothesis generating.

The study is limited to Caucasian subjects only. Genetic variation in telomere dynamics is likely to be effected by ethnicity. This will need to be considered when designing large-scale prospective studies in the future.

\section{CONCLUSION}

This study provides novel insights into genetic determination of telomere biology and its association with cardiac arrhythmia in ischaemic cardiomyopathy patients. Homozygosity for C-allele of SNP 12696304 significantly effects telomerase expression and its association with VA in this cohort. This suggests that variants in genes encoding TERC RNA subunit with known function in telomere biology have a 
functional effect and may modify clinical phenotype. Establishing longitudinal changes across genotypes in large-scale prospective studies will be required to investigate this further.

\section{FIGURE LEGEND}

Figure 1: Telomerase activity (mean $\pm S D)$ in cases and controls across different genotypes is shown here. The X-axis shows cases and controls in different genotypes (CC, CG, GG) of the SNP rs12696304 and the Y-axis shows mean telomerase activity.. For each genotype, individual data points for telomerase activity are shown separately for cases (circles) and controls (troangles). Means and SD intervals are shown. It is evident that telomerase activity was significantly different between cases and controls in C/C genotype only.

\section{ACKNOWLEDGEMENTS}

This study was facilitated by the Barts Health NHS Trust and NIHR Funded Barts Cardiovascular Biomedical Research Unit.

Author Contributions: VS concept \& design, patient recruitment \& lab work, data analyses \& interpretation, drafting article and revisions; SWB concept \& design, data analyses \& interpretation and critical revision of article; NGC data analyses \& interpretation and critical revision of article; SC data analyses \& interpretation; VB patient recruitment; $\mathbf{R H}$ critical revision of article; MD critical revision of article; AJ data analyses \& interpretation; ME critical revision of article; SS critical revision of article; KS concept \& design, data analyses \& interpretation and critical revision of article; RJS concept \& design, data analyses \& interpretation and critical revision of article 


\section{REFERENCES}

1. Moyzis RK, Buckingham JM, Cram LS, Dani M, Deaven LL, Jones MD, Meyne $\mathrm{J}$ et al. A highly conserved repetitive DNA sequence, (TTAGGG)n, present at the telomeres of human chromosomes. Proc Natl Acad Sci U S A 1998; 85: 6622-6626.

2. Harley $\mathrm{CB}$, Futcher $\mathrm{AB}$, Greider $\mathrm{CW}$. Telomeres shorten during ageing of human fibroblasts. Nature 1990; 345: 458-460.

3. Bendix L, Horn PB, Jensen UB, Rubelj I, Kolvraa S. The load of short telomeres, estimated by a new method, Universal STELA, correlates with number of senescent cells. Aging Cell 2010; 9: 383-397.

4. Olivieri F, Antonicelli R, Recchioni R, Mariotti S, Marcheselli F, Lisa R, Spazzafumo L et al. Telomere/telomerase system impairment in circulating angiogenic cells of geriatric patients with heart failure. Int J Cardiol 2013; 164(1): 99-105.

5. Samani NJ, Boultby R, Butler R, Thompson JR, Goodall, AH. Telomere shortening in atherosclerosis. Lancet 2001; 358: 472-473.

6. Brouilette S, Singh RK, Thompson JR, Goodall AH, Samani NJ. White cell telomere length and risk of premature myocardial infarction: Arterioscler Thromb Vasc Biol 2003; 23: 842-846.

7. Van der Harst P, Wong LS, de Boer RA, Brouilette, SW, van der steege G, Voors AA, Hall AS et al. Possible association between telomere length and renal dysfunction in patients with chronic heart failure. Am J Cardiol 2008; 102: $207-210$.

8. Collerton J, Martin-Ruiz C, Kenny A, Barrass K, von Zglincki T, Kirkwood T, Keavney B et al. Telomere length is associated with left ventricular function in the oldest old: the Newcastle 85+ study. Eur Heart J 2007; 28: 172-176. 
9. Salpea KD, Talmud PJ, Cooper JA, Maubaret CG, Stephens JW, Abelak K, Humphries SE et al. Association of telomere length with type 2 diabetes, oxidative stress and UCP2 gene variation. Atherosclerosis 2010; 209: 42-50.

10. Sawhney V, Campbell NG, Brouilette SW, Coppen SR, Harbo M, Baker V, Ikebe $\mathrm{C}$ et al. Telomere shortening and telomerase activity in ischaemic cardiomyopathy patients - potential markers of ventricular arrhythmia. Int $\mathrm{J}$ Cardiol 2016; 207: 157-163.

11. Vasa-Nicoter, $M$, Brouilette $S$, Mangino $M$, Thompson JR, Braund $P$, Clemitson JR, Mason A et al. (2005). Mapping of a major locus that determines telomere length in humans. Am J Hum Genet 2005; 76(1):147151.

12. Njajou OT, Cawthon RM, Damcott CM, Wu SH, Ott S, Garant MJ, Blackburn $\mathrm{EH}$ et al. Telomere length is paternally inherited and is associated with parental lifespan. Proc Natl Acad Sci U S A 2007; 104(29):12135-12139.

13. Slagboom PE, Droog S, and Boomsma DI. Genetic Determination of Telomere Size in Humans: A Twin Study of Three Age Groups. Am.J.Hum.Genet 1994b; 55: 876-882.

14. Andrew T, Aviv A, Falchi M, Surdulescu GL, Gardner JP, Lu X, Kimura M et al. Mapping genetic loci that determine leukocyte telomere length in a large sample of unselected female sibling pairs. Am J Hum Genet 2006; 78: 480486.

15. Okuda K, Bardeguez A, Gardner JP, Rodriguez P, Ganesh V, Kimura M, Skurnick J et al. Telomere length in the newborn. Pediatr Res 2002; 52: 377381.

16. Akkad A, Hastings R, Konje JC, Bell SC, Thurston H, Williams B. Telomere length in small-forgestational- age babies. BJOG 2006; 113: 318-323. 
17. Rufer N, Brummendorf TH, Kolvraa S, Bischoff C, Wadsworth L, Schulzer M et al. Telomere fluorescence measurements in granulocytes and $\mathrm{T}$ lymphocyte subsets point to a high turnover of hematopoietic stem cells and memory T cells in early childhood. J Exp Med. 1999; 190:157-167.

18. Frenck RW, Blackburn $\mathrm{EH}$, and Shannon KM. The rate of telomere sequence loss in human leukocytes varies with age. Proc.Natl.Acad.Sci.USA. 1998; 95: $5607-5610$.

19. Aviv A. Genetics of leukocyte telomere length and its role in atherosclerosis. Mutat Res. 2012; 730(1-2): 68-74.

20. Codd V, Mangino M, van der Harst P, Braund PS, Kaiser M, Beveridge AJ, Rafelt $\mathrm{S}$ et al. Common variants near TERC are associated with mean telomere length. Nat Genet. 2010; 42(3): 197-199.

21. Mukherjee M, Brouilette S, Stevens S, Shetty KR, Samani NJ. Association of shorter telomeres with coronary artery disease in Indian subjects. Heart. 2009; 95(8): 669-673.

22. Cawthon RM. Telomere measurement by quantitative PCR. Nucleic Acids Res 2002; 30(10): e47.

23. Brouilette SW, Moore JS, McMahon AD, Thompson JR, Ford I, Shepherd J, Packard CJ et al. Telomere length, risk of coronary heart disease, and statin treatment in the West of Scotland Primary Prevention Study: a nested casecontrol study. Lancet 2007; 369:107-114.

24. Cawthon RM. Telomere length measurement by a novel monochrome multiplex quantitative PCR method. Nucleic Acids Res 2009; 37(3): e21.

\section{Roche TeloTAGGG Telomerase PCR ELISA.} http://lifescience.roche.com/shop/products/telotaggg-telomerase-pcr-elisa 26. Levy D, Neuhausen SL, Hunt SC, Kimura M, Hwang SJ, Chen W, Bis JC et al. Genome-wide association identifies OBFC1 as a locus involved in human 
leukocyte telomere biology. Proc. Natl. Acad. Sci. USA. 2010; 107:92939298.

27. Egan ED, Collins K. An enhanced H/ACA RNP assembly mechanism for human telomerase RNA. Mol. Cell. Biol. 2012; 32: 2428-2439.

28. Miyake Y, Nakamura M, Nabetani A, Shimamura S, Tamura M, Yonehara S, Saito M et al. RPA-like mammalian Ctc1-Stn1-Ten1 complex binds to singlestranded DNA and protects telomeres independently of the Pot1 pathway. Mol. Cell. 2009; 36:193-206.

29. Ding H, Schertzer M, Wu X, Gertsenstein M, Selig S, Kammori M, Porvali et al. Regulation of murine telomere length by Rtel1: an essential gene encoding a helicase-like protein. Cell. 2004; 117:873-886.

30. Barber LJ, Youds JL, Ward JD, Mcllwraith MJ, O’Neill NJ, Petalcorin MI, Martin SJ et al. RTEL1 maintains genomic stability by suppressing homologous recombination. Cell. 2008; 135: 261-271. 


\section{TABLES}

Table 1: Baseline demographics of cases and controls included in the study

\begin{tabular}{|c|c|c|c|}
\hline $\mathbf{N}=\mathbf{9 0}$ & Cases & Controls & p-value \\
\hline Number of patients & 35 & 55 & 0.19 \\
\hline Age(yrs), mean \pm SD & $69.3 \pm 7.6$ & $71.6 \pm 8.8$ & 0.43 \\
\hline White cell count (109/L), & $7.7 \pm 1.7$ & $7.46 \pm 1.7$ & 0.78 \\
mean \pm SD & & & 0.28 \\
\hline Male gender,n(\%) & $32(91.4)$ & $48(87.3)$ & 0.77 \\
\hline Diabetes,n(\%) & $7(20)$ & $18(32.7)$ & 0.18 \\
\hline Hypertension,n(\%) & $14(40)$ & $25(45.4)$ & 0.75 \\
\hline Follow-up since ICD implant, & $46.5 \pm 26.9$ & $38.1 \pm 30.5$ & 0.49 \\
\hline months;mean \pm SD & & & 0.62 \\
\hline LVEF,\%;mean \pm SD & $26.73 \pm 7.2$ & $27.24 \pm 7.6$ & $60.59 \pm 20.22$ \\
\hline eGFR, mean \pm SD & $63.94 \pm 21.8$ & $50(90.91)$ & \\
\hline NYHA GradeIII, n(\%) & $33(94.28)$ & & \\
\hline
\end{tabular}

[Results shown as meantstandard deviation or number of patients and \% of total group. LVEF: Left Ventricular Ejection Fraction, eGFR: Glomerular Filteration Rate, NYHA: New York Heart Association classification] 
Table 2: Multivariate analysis of Potentially-fatal VA and Genotype

\begin{tabular}{|c|c|c|c|}
\hline Parameter & Odds Ratio & $\begin{array}{c}\mathbf{9 5 \%} \text { Wald } \\
\text { Confidence Limits }\end{array}$ & p-value \\
\hline SNP C/C & 1 & - & - \\
\hline SNP C/G & 0.632 & $0.211-1.891$ & 0.48 \\
\hline SNP G/G & 0.921 & $0.194-4.374$ & 0.84 \\
\hline Gender & 0.845 & $0.138-5.158$ & 0.85 \\
\hline Age & 0.938 & $0.885-0.993$ & 0.03 \\
\hline
\end{tabular}


$\underline{\text { Table 3: Potentially-fatal VA and mean telomere length in different genotypes }}$ of SNP rs12696304

\begin{tabular}{|c|c|c|c|}
\hline Parameter & Odds ratio & $\begin{array}{c}\text { 95\% Wald } \\
\text { Confidence Limits }\end{array}$ & \\
\hline Mean telomere length in & 1.06 & $0.86-1.32$ & 0.58 \\
C/C genotype & & & \\
\hline Mean telomere length in & 0.91 & $0.57-1.44$ & 0.68 \\
C/G genotype & & & \\
\hline Mean telomere length in & 0.92 & $0.55-1.52$ & 0.73 \\
G/G genotype & & & \\
\hline
\end{tabular}


Table 4: Potentially-fatal VA and telomerase activity in different genotypes of $\underline{\text { SNP } r s 12696304}$

\begin{tabular}{|c|c|c|c|}
\hline Parameter & Odds Ratio & 95\% Wald & p-value \\
& & Confidence & \\
\hline Telomerase activity in & 7.507 & $1.00-56.61$ & 0.04 \\
C/C genotype & & & \\
\hline Telomerase activity in & 2.759 & $0.352-21.60$ & 0.33 \\
C/G genotype & & & 0.43 \\
\hline Telomerase activity in & 4.137 & $0.117-145.91$ & \\
G/G genotype & & & \\
\hline
\end{tabular}


Figure 1: Telomerase activity (mean \pm SD) in cases and controls across different genotypes

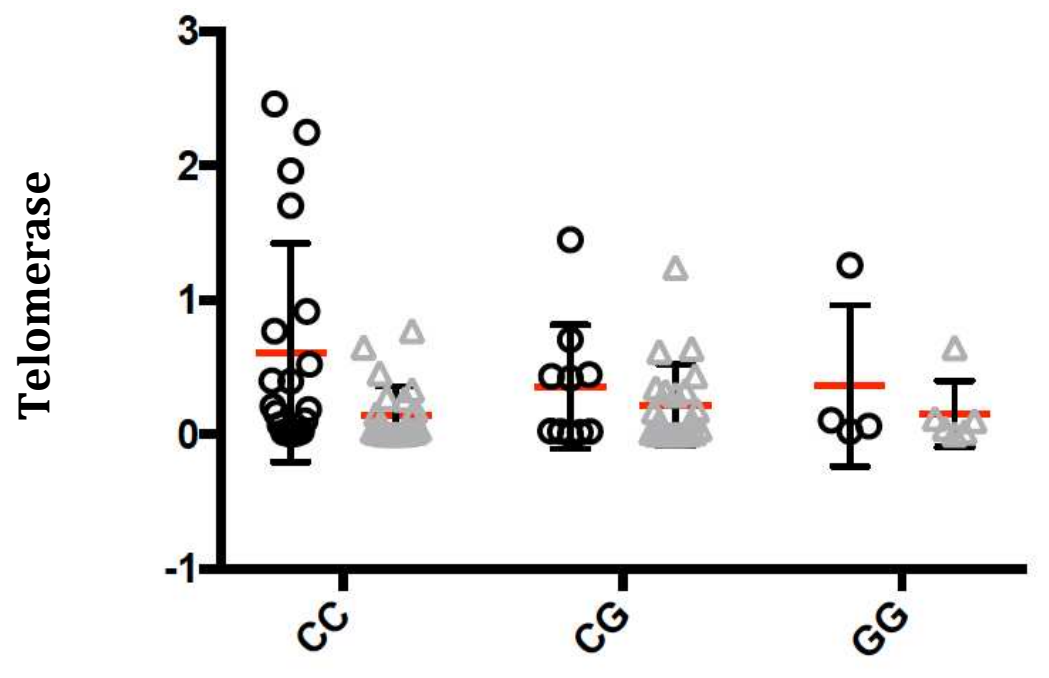

- CASES

$\triangle$ CONTROLS

Genotype 